\title{
New results on OGLE Cepheids and PL relations in the LMC and SMC
}

\author{
A. Udalski \\ Warsaw University Observatory, Al. Ujazdowskie 4, 00-478 Poland
}

\begin{abstract}
We present results of the search for pulsating variable stars in the Magellanic Cloud fields covering central parts of these galaxies. The data were collected during the second phase of the Optical Gravitational Lensing Experiment survey (OGLE-II) from 1997 to 2000. In total, several thousand pulsating stars (Cepheids, RR Lyr) were found in both Magellanic Clouds. The photometric data of all objects are available to the astronomical community from the OGLE Internet archive. We present basic properties of pulsating stars in the Magellanic Clouds including Period-Luminosity relations for Cepheids. We also discuss observational prospects for the pulsating star field in the ongoing third phase of the OGLE project (OGLE-III) which started in 2001.
\end{abstract}

\section{The OGLE survey}

The Optical Gravitational Lensing Experiment (OGLE) is a long term, large scale photometric survey with more than a decade long history. It started in 1992 April as one of the first large microlensing surveys.

Historically the OGLE project is divided into three phases. Each of the phases is connected with observing capabilities of the OGLE survey. Transition to the next phase was always related with large hardware and software upgrade increasing the observing capabilities by an order of magnitude or more.

The first phase of the OGLE project - OGLE-I - began in 1992. During this phase only selected fields in the Galactic bulge were observed for about 70 nights per year. The 1-m Swope telescope at the Las Campanas Observatory in Chile, operated by the Carnegie Institution of Washington, equipped with $2048 \times 2048$ thick CCD camera was used. The main scientific contribution of this phase included the first works in the new gravitational microlensing field: discovery of the first microlensing events toward the Galactic center; first binary microlensing; and first determination of the empirical optical depth to microlensing toward the Galactic bulge. The OGLE-I phase lasted up to 1995 September.

In 1997 January, after one year break, the second phase - OGLE-II - began. The upgrade included a new 1.3-m Warsaw telescope, dedicated for the project, and new "first generation" $2048 \times 2048$ thin CCD camera working in drift-scan mode. Year-round access to the telescope and a more efficient detector increased the data flow of the project by a factor of 30 . New targets, namely the Magellanic Clouds, became accessible to OGLE-II. Beside much more extended work in the 
microlensing field, the main scientific contribution of this phase was the discovery of hundreds of thousands of variable stars and the precise and well-calibrated photometry of millions of stars from such interesting regions of the sky as the Galactic bulge and Magellanic Clouds. Results of this phase are presented in this paper.

OGLE-II ended in 2000 November. During the next months another major hardware upgrade took place - the "first generation" CCD camera was replaced with a new eight-chip $8192 \times 8192$ mosaic. Observing capabilities of the project increased by another order of magnitude. Regular observations - OGLE-III phase - were started in 2001 June. Currently, OGLE-III is a Terabyte survey with the data flow of about $3.5 \mathrm{~TB} / \mathrm{yr}$. Regularly about 200 million stars in the Galactic bulge, 27 million in the LMC and 5 million in the SMC are observed. Photometry of these stars is obtained and analyzed in real time at the telescope. Although the OGLE-III survey is still in its early phases, many new discoveries have already been announced, including discovery of the first extrasolar planets detected with the photometric transit method.

A large fraction of the photometric data collected during the OGLE survey is available to the astronomical community from the following WWW addresses:

$$
\begin{gathered}
\text { http://ogle.astrouw.edu.pl/ } \\
\text { http://bulge.princeton.edu/ }{ }^{\sim} \text { ogle }
\end{gathered}
$$

where one can also find all new information on the current project status and developments.

\section{Pulsating stars in the OGLE-II fields}

Thousands of pulsating stars were discovered in the OGLE-II data. We list below the main catalogs released by the OGLE collaboration:

\section{Classical Cepheids}

- Classical Cepheids in the LMC: $\approx 1300$ objects (Udalski et al. 1999c)

- Classical Cepheids in the SMC: $\approx 2000$ objects (Udalski et al. 1999d)

- Double Mode Cepheids in the MC: 95 objects in the SMC (Udalski et al. 1999a) and 81 in the LMC (Soszyński et al. 2000)

- Second Overtone Cepheids in the SMC: 13 objects (Udalski et al. 1999b)

- Classical Cepheids in IC 1613: $\approx 140$ objects (Udalski et al. 2001)

\section{Population II Cepheids}

- Population II Cepheids in the LMC: $\approx 40$ objects (Udalski et al. 1999c)

- Population II Cepheids in the SMC: several objects (Udalski et al. 1999d)

- Population II Cepheids in the Galactic bulge: $\approx 50$ objects (Kubiak \& Udalski 2003) 


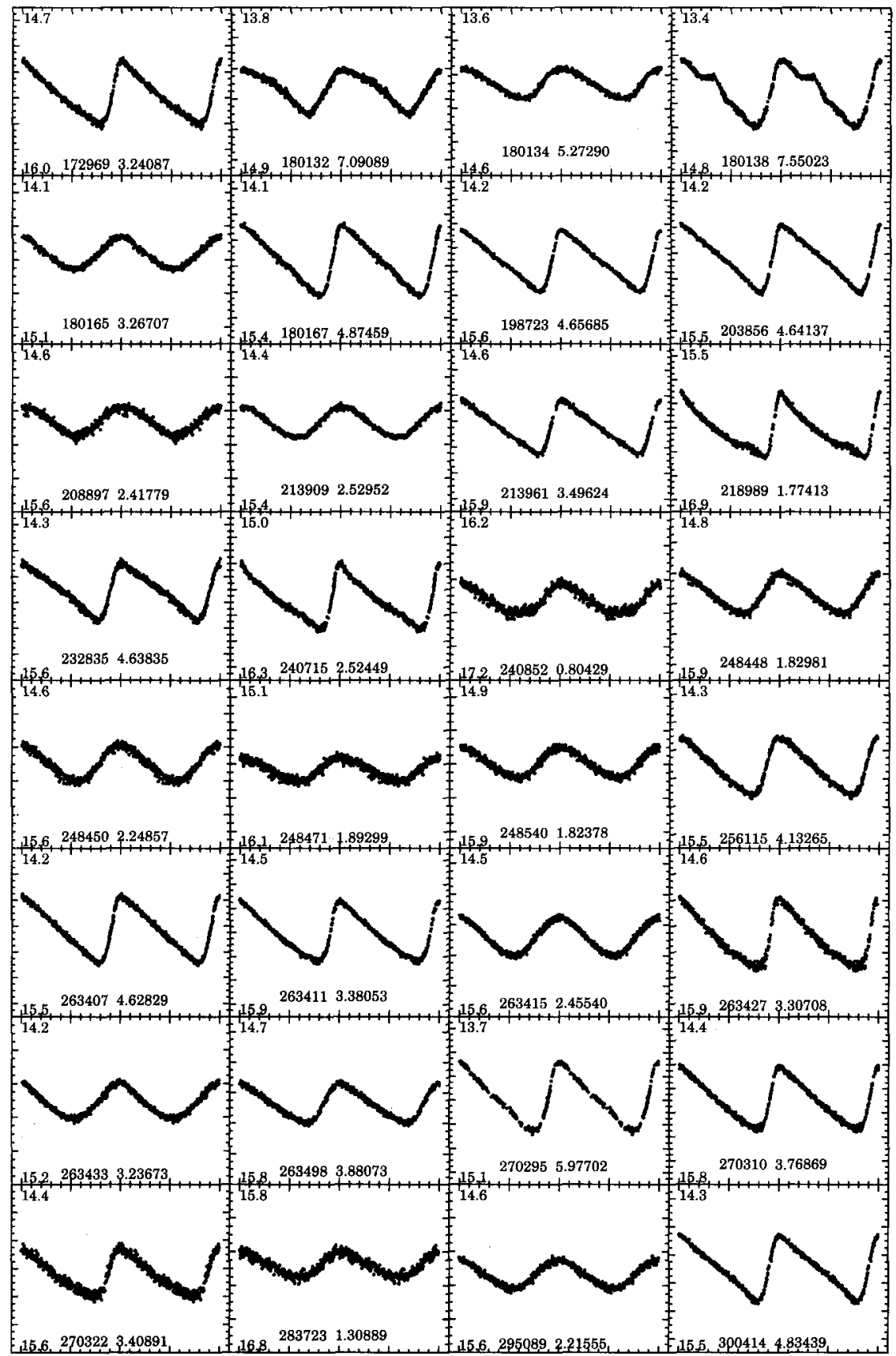

Figure 1. Sample of Cepheids from the LMC_SC2 field in the LMC. 
OGLE052218.07-692827.4

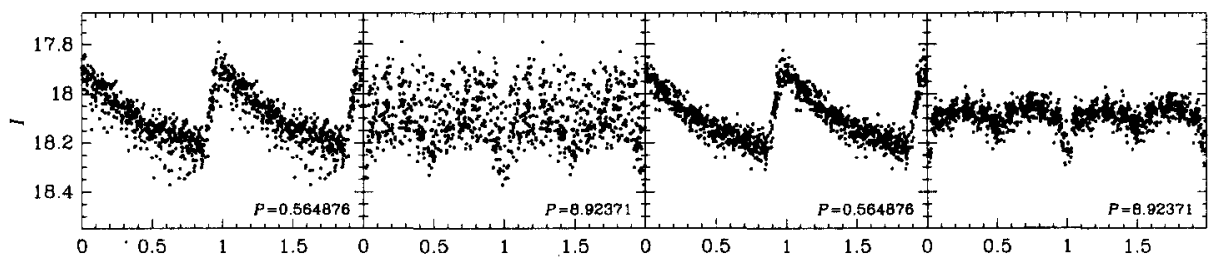

OGLE051822.60-691817.3
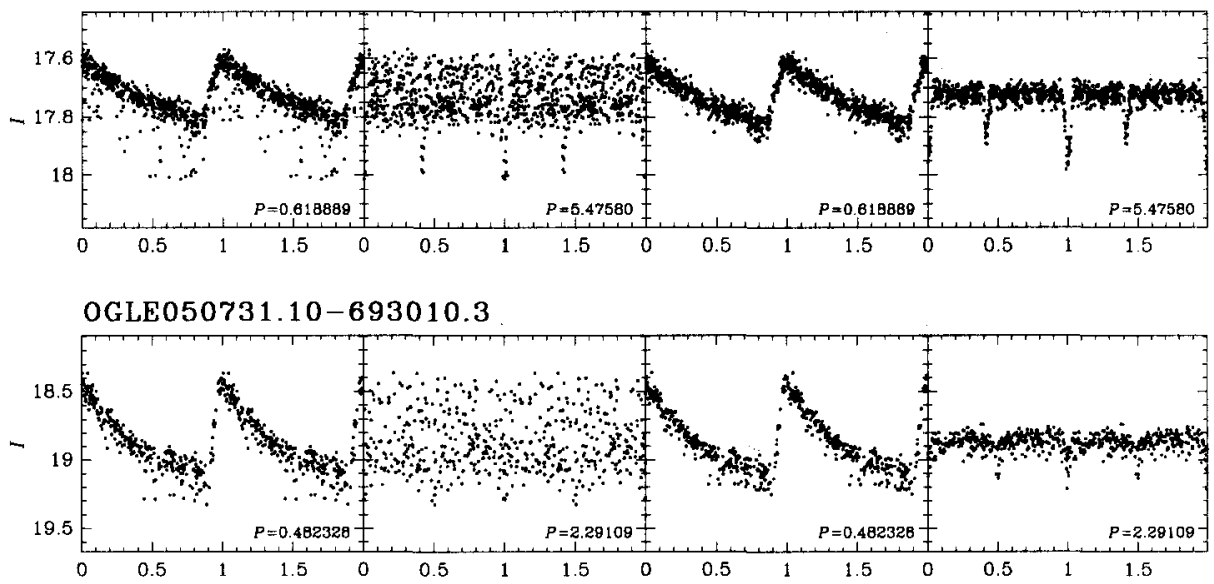

Figure 2. RR Lyr stars with simultaneous eclipsing variability.

\section{RR Lyr stars}

- RR Lyrae in the LMC: $\approx 7600$ objects (Soszyński et al. 2003)

- RR Lyrae in the SMC: $\approx 570$ objects (Soszyński et al. 2002)

- RR Lyrae in the Galactic bulge: $\approx 2000$ objects (not released yet)

- RR Lyrae in the Carina Dwarf Galaxy: $\approx 30$ objects (Udalski 2000).

\section{Other stars}

- $\beta$ Cephei stars (Pigulski \& Kołaczkowski 2002)

- Pulsating Giants (Soszyński 2003, in preparation, Kiss \& Bedding 2003)

Fig. 1 presents a sample of light curves of Classical Cepheids from the LMC. Fig. 2 shows three RR Lyr stars from the LMC which simultaneously show eclipsing binary variability (Soszyński et al. 2003). If future observations confirm that these systems are physically bound, and not optical blends, precise empirical determination of parameters of RR Lyr components will be possible for the first time. 
Table 1. Slope of the P-L relations of fundamental mode Cepheids

\begin{tabular}{lcccc}
\hline Object & $B$ & $V$ & $I$ & $W_{I}$ \\
\hline LMC & $-2.44 \pm 0.05$ & $-2.78 \pm 0.03$ & $-2.98 \pm 0.02$ & $-3.31 \pm 0.01$ \\
& $\sigma=0.24$ & $\sigma=0.16$ & $\sigma=0.11$ & $\sigma=0.06$ \\
SMC & $-2.21 \pm 0.05$ & $-2.57 \pm 0.04$ & $-2.84 \pm 0.03$ & $-3.31 \pm 0.02$ \\
& $\sigma=0.32$ & $\sigma=0.25$ & $\sigma=0.20$ & $\sigma=0.11$ \\
IC 1613 & - & $-2.76 \pm 0.10$ & $-2.95 \pm 0.07$ & $-3.26 \pm 0.06$ \\
& - & $\sigma=0.19$ & $\sigma=0.15$ & $\sigma=0.11$ \\
NGC 6822 & - & $-2.61 \pm 0.12$ & $-2.88 \pm 0.07$ & $-3.30 \pm 0.09$ \\
\hline \hline
\end{tabular}

\section{Period-Luminosity relations for Classical Cepheids}

Table 1 contains slopes of the period-luminosity (P-L) relation of Classical Cepheids pulsating in the fundamental mode obtained from the OGLE-II data. We additionally included there results for the galaxy NGC6822 (Pietrzyński 2003, private communication). Table 2 contains the slopes for the first overtone Cepheids from the Magellanic Clouds.

Table 2. Slope of the P-L relation of first overtone mode Cepheids

\begin{tabular}{ccccc}
\hline Object & $B$ & $V$ & $I$ & $W_{I}$ \\
\hline LMC & $-3.23 \pm 0.07$ & $-3.33 \pm 0.05$ & $-3.37 \pm 0.04$ & $-3.41 \pm 0.02$ \\
& $\sigma=0.26$ & $\sigma=0.19$ & $\sigma=0.13$ & $\sigma=0.06$ \\
SMC & $-2.96 \pm 0.06$ & $-3.09 \pm 0.05$ & $-3.30 \pm 0.04$ & $-3.57 \pm 0.02$ \\
& $\sigma=0.32$ & $\sigma=0.26$ & $\sigma=0.20$ & $\sigma=0.12$ \\
\hline \hline
\end{tabular}

The zero points of the $\mathrm{P}-\mathrm{L}$ relation are the subject of long-lasting discussions. A definitive answer will probably be provided by the future space astrometric missions in the time scale of a decade. One should, however, remember that the OGLE-II observations of several stellar standard candles in the same galaxies provide strong constraints on calibration of these standard candles. The differential analysis is basically free from systematic errors, such as the uncertainty of interstellar extinction and the uncertainty of the zero point of photometry. Table 3 contains the differences of brightness of a 10-d Cepheid (CEP) and other standard candles, namely the tip of the red giant stars (TRGB), RR Lyr stars (RR) and red clump (RC) stars in the Magellanic Clouds and galaxies IC 1613 and NGC 6822 (Pietrzyński 2003, private communication). Fig. 3 shows the difference of brightness of Cepheids and TRGB as a function of metallicity of Cepheids in the presented galaxies.

Similar values of differences of brightness of stellar standard candles in so different galaxies (Fig. 3) indicate that the zero points of the $\mathrm{P}-\mathrm{L}$ relation of Classical Cepheids are independent or marginally dependent on properties of the environment, i.e. metallicity. Table 1 indicates that the same conclusion can be drawn for slopes of the P-L relation, in particular for the I-band or $W_{I}$ index. Table 3 also provides constraints on calibration of stellar standard 


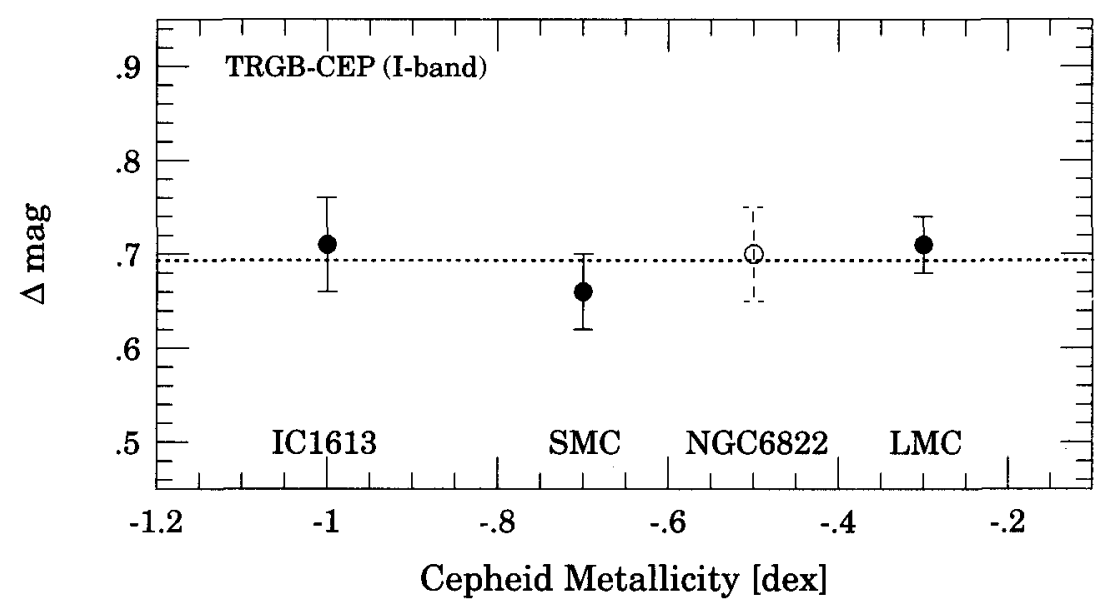

Figure 3. Difference of brightness of 10-d Cepheids and TRGB stars in different galaxies as a function of the metallicity of Cepheids.

candles. Independent calibrations of these candles must provide differences of brightness in agreement with the observed values presented in Table 3.

Table 3. Constraints on zero points of standard candle calibration

\begin{tabular}{lccc}
\hline Object & CEP-TRGB & CEP-RR & CEP-RC \\
& $I$ & $V$ & $I$ \\
\hline LMC & $-0.71 \pm 0.03$ & $-4.62 \pm 0.03$ & $-4.33 \pm 0.03$ \\
SMC & $-0.66 \pm 0.04$ & $-4.61 \pm 0.04$ & $-4.25 \pm 0.04$ \\
IC 1613 & $-0.71 \pm 0.05$ & - & - \\
NGC 6822 & $-0.70 \pm 0.05$ & - & - \\
\hline \hline
\end{tabular}

\section{Future prospects}

The OGLE-III projects which began regular observations of the Magellanic Clouds in June 2001 is still in the early phases. Nevertheless, to the end of June 2003 about 100-250 epochs were already collected for the LMC fields and about 200 epochs for the SMC fields. The LMC and SMC fields now cover practically the entire galaxies (Fig. 4). Photometry of more than 30 million stars from both MCs is obtained in the real time. The new data pipeline system based on the image subtraction technique provides now photometry with mmag accuracy for the brightest stars. We believe that in the time scale of a few years the vast majority of pulsating stars from the Magellanic Clouds down to $\approx 20$ mag will be detected by the OGLE-III survey and photometry will be released to the astronomical community.

Acknowledgments. The paper was partly supported by the Polish KBN grant 2P03D02124 to A. Udalski. Partial support for the OGLE project was 


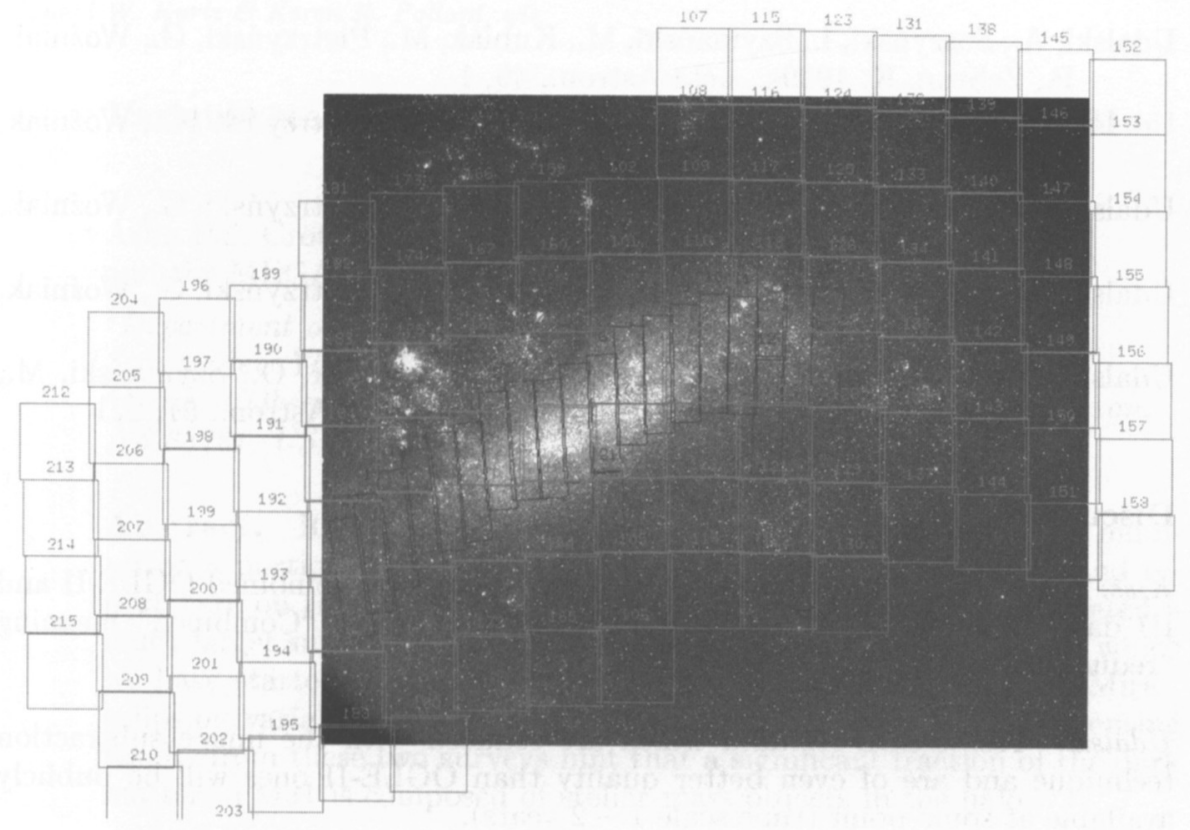

Figure 4. OGLE-II (black rectangles) and OGLE-III (grey squares) field contours plotted on the DSS image of the LMC.

provided with the NSF grants AST-9820314 and AST-0204908 and NASA grant NAG5-12212 to B. Paczyński. We acknowledge usage of The Digitized Sky Survey which was produced at the Space Telescope Science Institute based on photographic data obtained using The UK Schmidt Telescope, operated by the Royal Observatory Edinburgh.

\section{References}

Kiss, L.L., Bedding, T.R. 2003, MNRAS, 343, 79

Kubiak, M., Udalski A. 2003, Acta Astron., 53, 117

Pigulski, A., Kołaczkowski, Z. 2002, A\&A, 388, 88

Soszyński, I., Udalski, A., Szymański, M., Kubiak, M., Pietrzyński, G., Woźniak, P., Zebruń K. 2000, Acta Astron., 50, 451

Soszyński, I., Udalski, A., Szymański, M., Kubiak, M., Pietrzyński, G., Woźniak, P., Żebruń, K., Szewczyk, O., Wyrzykowski, L. 2002, Acta Astron., 52,369

Soszyński, I., Udalski, A., Szymański, M., Kubiak, M., Pietrzyński, G., Woźniak, P., Żebruń, K., Szewczyk, O., Wyrzykowski, L. 2003, Acta Astron., 53, 93

Udalski A. 2000, Acta Astron., 50, 279 
Udalski, A., Soszyński, I., Szymański, M., Kubiak, M., Pietrzyński, G., Woźniak, P., Żebruń, K. 1999a, Acta Astron., 49, 1

Udalski, A., Soszyński, I., Szymański, M., Kubiak, M., Pietrzyński, G., Woźniak, P., Żebruń, K. 1999b, Acta, Astron., 49, 45

Udalski, A., Soszyński, I., Szymański, M., Kubiak, M., Pietrzyński, G., Woźniak, P., Zebruń, K. 1999c, Acta Astron., 49, 223

Udalski, A., Soszyński, I., Szymański, M., Kubiak, M., Pietrzyński, G., Woźniak, P., Żebruń, K. 1999d, Acta Astron., 49, 437

Udalski, A., Wyrzykowski, Ł., Pietrzyński, G., Szewczyk, O., Szymański, M., Kubiak, M., Soszyński, I., Żebruń, K. 2001, Acta Astron., 51, 221

\section{Discussion}

Kiss: For red giant stars, it would be essential to have combined OGLE-II and III data. Can we expect such a database and when? ("Combined" meaning "reduced in the same fashion").

Udalski: Yes, OGLE-III data which are reduced with the image subtraction technique and are of even better quality than OGLE-II ones will be publicly available at some point (time scale $1-2$ years).

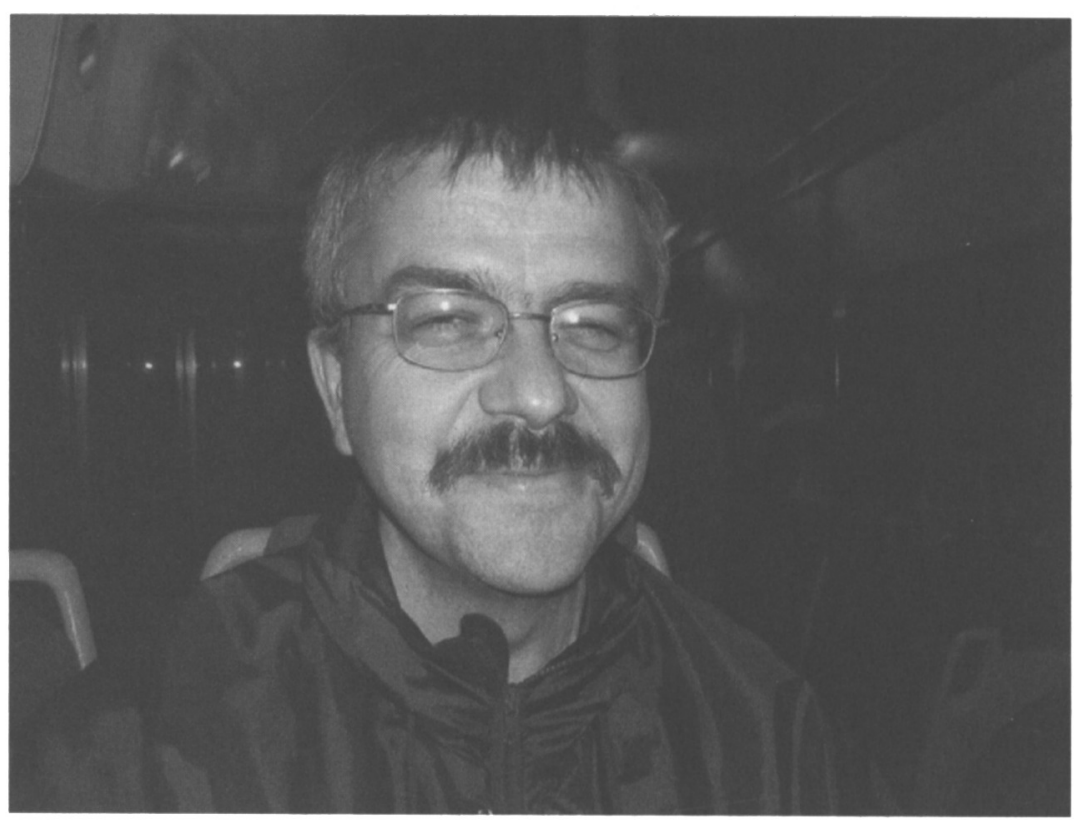

Andre Udalski 